\title{
DEVELOPMENT OF A COLOR DETECTION AND ANALYZING SYSTEM
}

\author{
Shubham Kumar ${ }^{1}$, Dasari Karthik ${ }^{2}$, Pradeep Khanna ${ }^{3}$ \\ ${ }^{1}$ Student, Netaji Subhas Institute Of Technology (DELHI) \\ ${ }^{2}$ Student, Netaji Subhas Institute Of Technology (DELHI) \\ ${ }^{3}$ Associate Professor, Department Of MPAE, Netaji Subhas Institute Of Technology
}

\begin{abstract}
Color measurements have traditionally been linked to expensive and difficult to handle equipment. This usually requires a thorough knowledge of color spaces, colorimetric transformations and color management systems. The TCS3200CS color sensor (I2C Sensor Color Grove), a system for capturing, processing and color management that allows the colors of any non-selfluminous object using a low-cost hardware based on Arduino, is presented in this paper. Thus, a prototype has been developed to sense the sample color and estimate the percentage of color component colors in it. The developed system is based on an RGB color sensor with photo detectors, applied to color detection of paint. The results show acceptable accuracy values.
\end{abstract}

Keywords: Arduino UNO, TCS3200 Color Sensor, LCD Module, RGB LED, Potentiometer, Jumper Wires

\section{INTRODUCTION}

Mixing of primary colors in various proportions to get different shades have been a requirement and curiosity from the very beginning. With the increase in infrastructural growth and technological advancements, the choices for diversified color shades also increased. Survey into this field has indicated that earlier creating different shades of colors was done using the experience of the mixing personnel later, sophisticated machines were developed where a library of large number of shades are shown in the memory. The machine is capable of telling the proportion of color components colors in that shade taking one base color to be mixed with specified amount of strainer. The manufacturer decides these combinations for the fixed quantity of the required shades. The limitation of this system is that a shade belonging the library of shades can only be made and that too in a fixed quantity. The present work is based on three primary colors Red, Green and Blue and is based upon sensing the given shade with the help of TCS 3200 Color Sensor [1]. This system uses Arduino Uno R3 as a microprocessor. The sensor senses the color and sends this information to the microprocessor, which as per the programming code interprets the input from the sensor and gives the output in the form of different intensities of primary colors, which if mixed, will give the color sensed by the sensor. It can be useful for color identification and detection for food-processing units, color printer applications, paint-mixing applications and other industrial applications including robotics [2].

\section{WORKING METHODOLOGY}

In this project, Color Sensor TCS3200 module is used for detecting the percentage of primary colors (red, green and blue, or RGB) colors that are physically available in a RGB
LED and that will be displayed on a 16X2 LCD Module. We can display primary colors and generate specific colors on RGB LED by modifying the Arduino code. The project demonstrates the basic interfacing of TCS3200 color sensor, Arduino Uno, RGB LED and LCD Module. When a colored object is lighted with white light, it reflects only fractions of the primary colors of the incident light i.e. R, G and B [3].

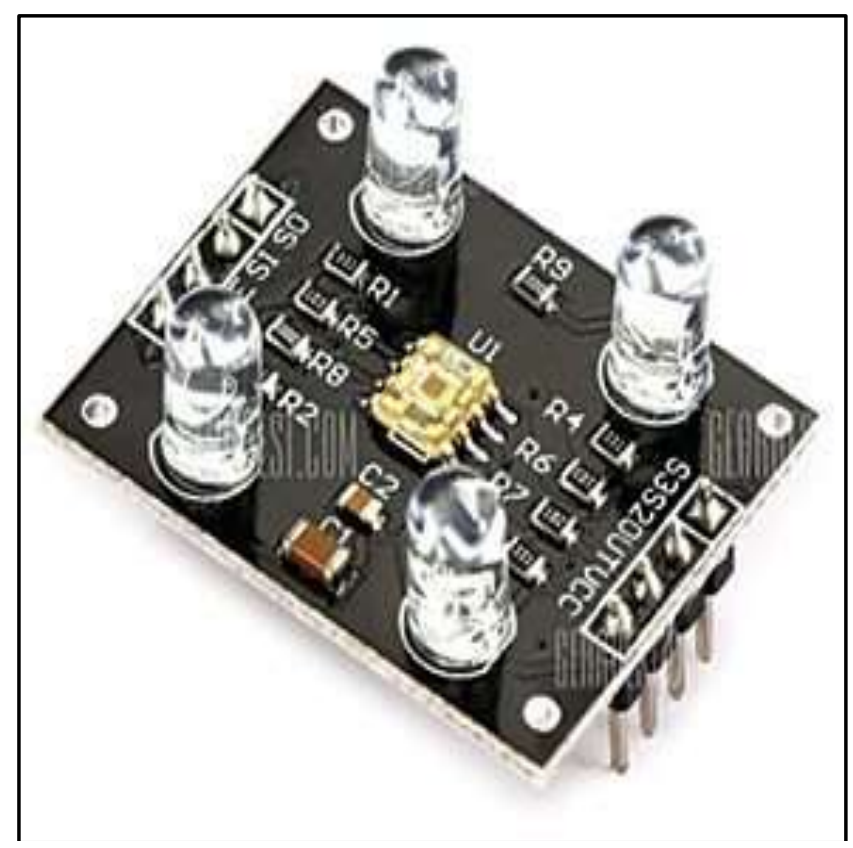

Fig 1: TCS3200 color sensor module [4]

TCS3200 color sensor module is shown in Fig. 1 and microscopic view of the RGB arrays is shown in Fig. 2. On the microscopic level, you can see the square boxes inside the eye on the sensor. These square boxes are arrays of the 
RGB matrix as shown in Fig. 3. Each of these boxes have four types of photodiodes sensitive to red, blue, green and white light and four white led for lighting the object. This sensor can be used to identify any number of colors with an accurate programming code [5].

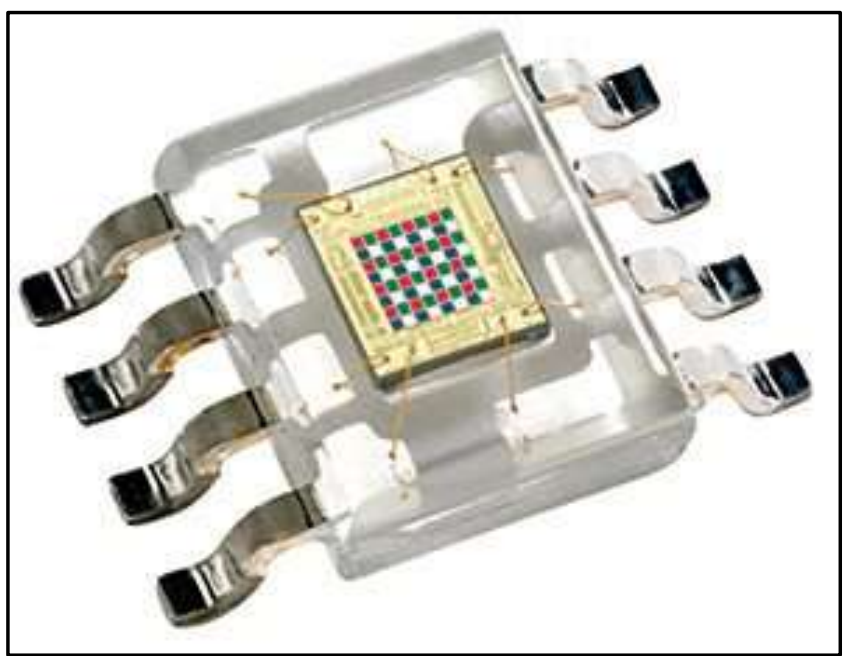

Fig 2: Microscopic view of TCS3200 chip [4]
TCS3200 module has eight pins as shown in Fig. 4. This module consists of programmable color light-to-frequency converters that combine configurable silicon photodiodes and current-to-frequency converter on a single monolithic CMOS integrated circuit. Each sensor array in these three arrays is selected separately, depending on the requirement. Hence, it is known as a programmable sensor. Output is square-wave (50 per cent duty cycle) with frequency directly proportional to light intensity (irradiance). As shown in Fig. 5.

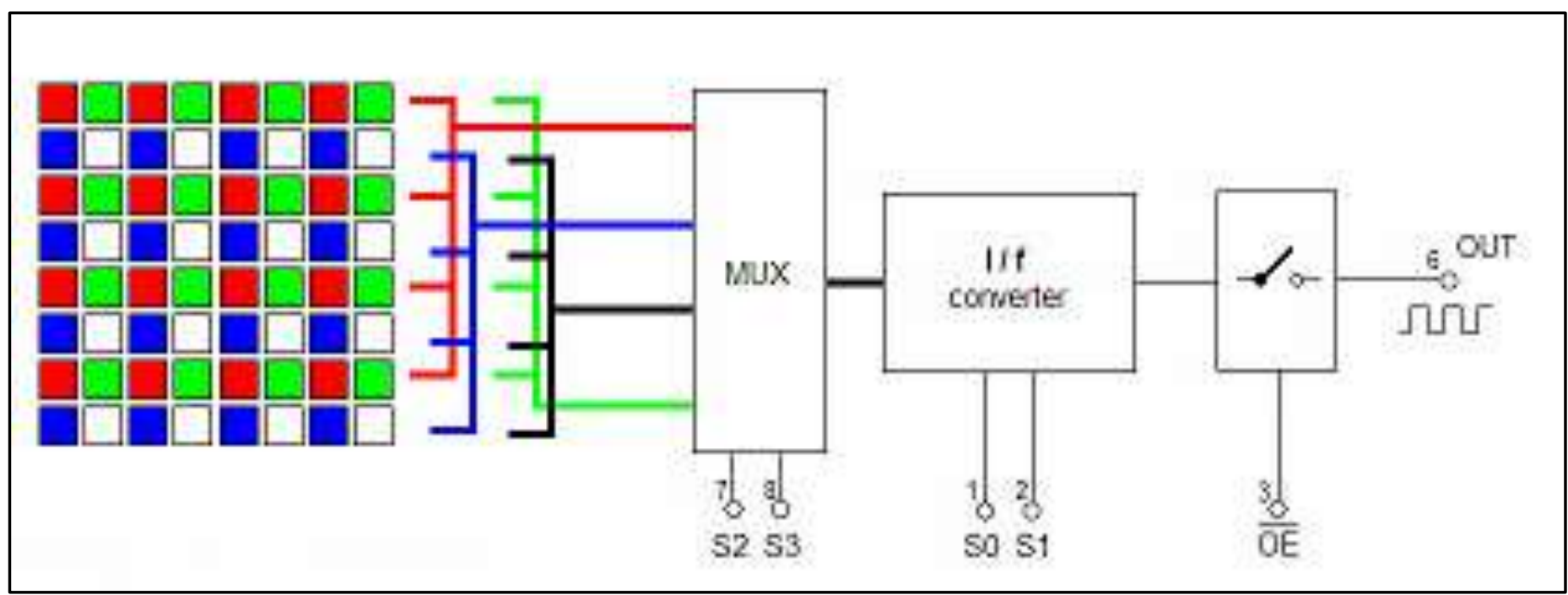

Fig 3: Microscopic view of RBG Matrix Array [6]

Digital inputs and outputs allow direct interface to the MCU or other logic circuitry. Output enable (OE) places the output in high-impedance state for multiple units sharing an MCU input line. In TCS3200, the light-to-frequency converter reads an $8 \times 8$ array of photodiodes. Sixteen photodiodes have blue filters, another sixteen have green, yet another sixteen have red and remaining sixteen are clear with no filters. All photodiodes of the same color are connected in parallel [7]. 


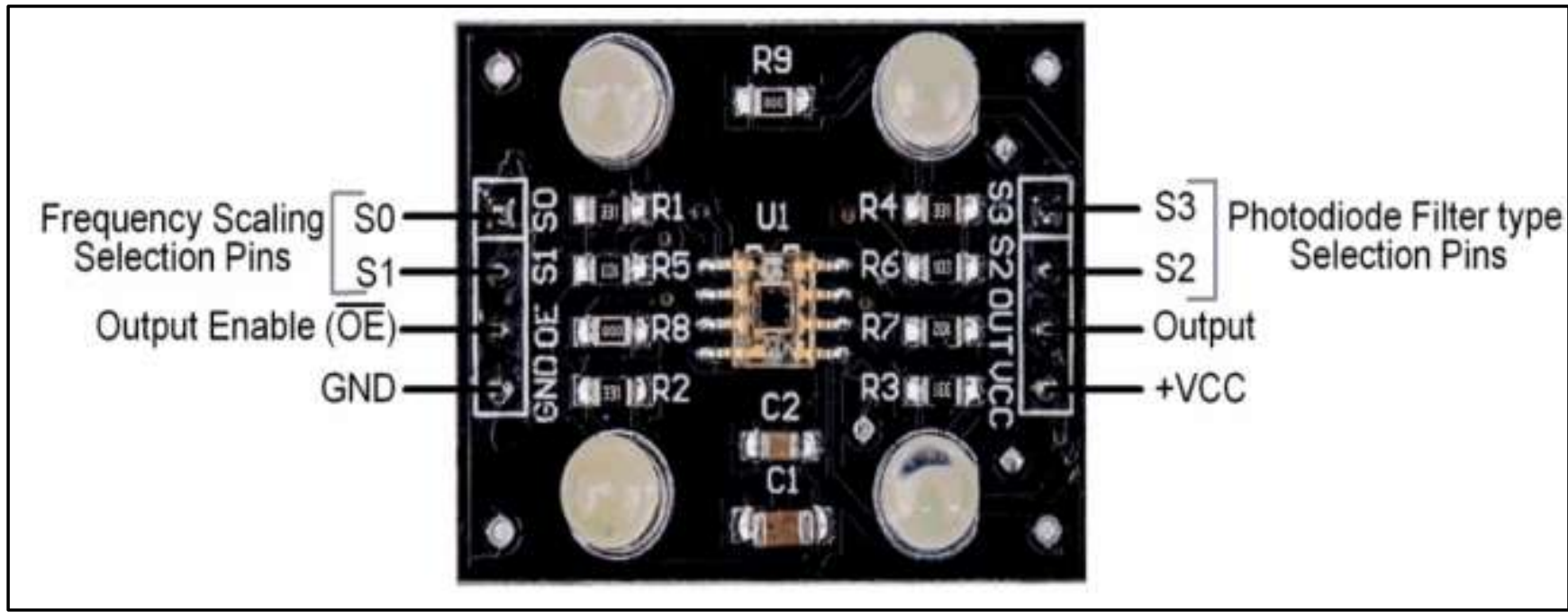

Fig 4: Color Sensor TCS3200 Pin Description [4]

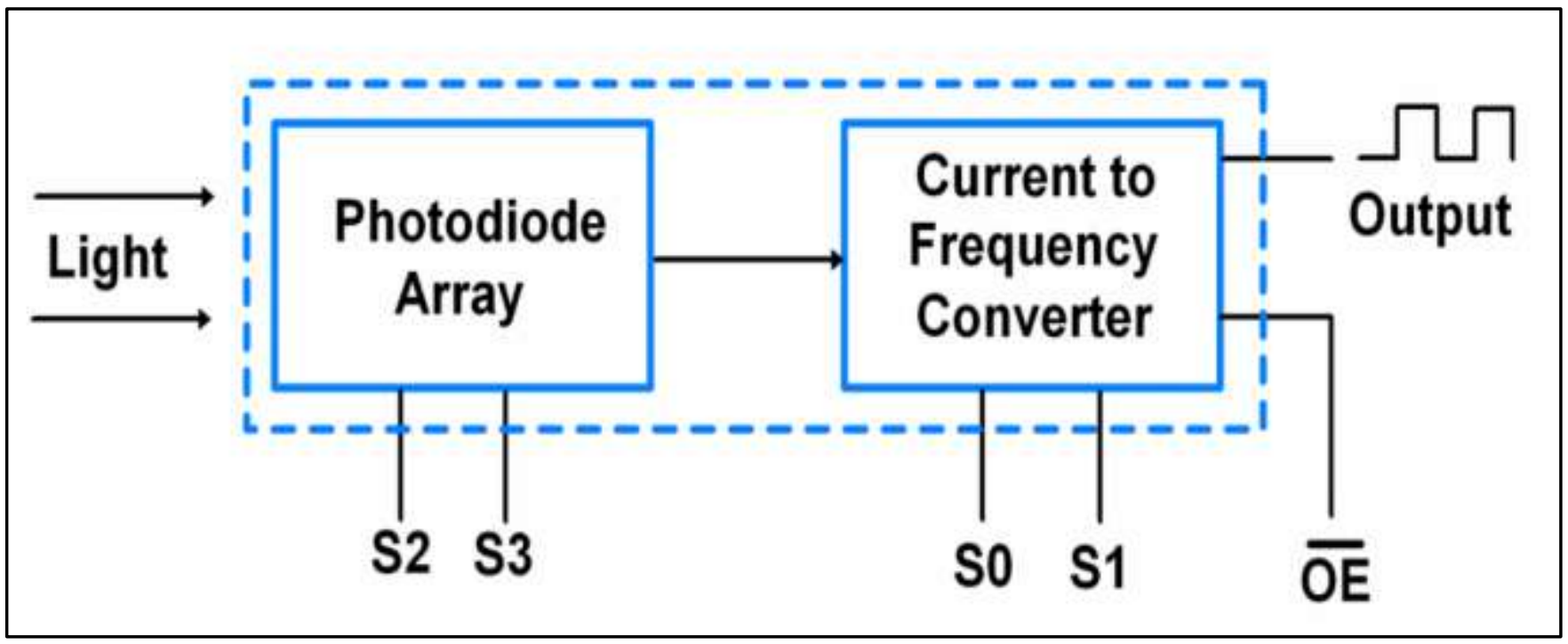

Fig 5: Functional block diagram of Color Sensor [4]

Detecting the color of a given object means to establish the values of these fractions. For this purpose, the object is lighted with white light and then we measure separately the intensity of the red, blue and green radiations reflected by the object. We can activate a desired type of photodiode applying TTL signals to the entries S2 and S3 as shown in Table 1[8].

So we can separately record the component R, G \& B of the light reflected by the object. On the output of TCS 3200 we don't obtain an analog signal, but a square wave signal with the frequency determined by the intensity of the light received by the sensor. The range of the typical output frequency is $2 \mathrm{~Hz} \sim 500 \mathrm{KHz}$. We can chose the range of the output frequency by different combinations of inputs pins S0 and S1, as shown in Table2[9].
Table 1: Photodiode type selection

\begin{tabular}{|l|l|l|}
\hline S2 & S3 & $\begin{array}{l}\text { Photodiode Type with their } \\
\text { respective Filter }\end{array}$ \\
\hline 0 & 0 & Red \\
\hline 0 & 1 & Blue \\
\hline 1 & 0 & Clear \\
\hline 1 & 1 & Green \\
\hline
\end{tabular}

Table 2: Frequency Scaling

\begin{tabular}{|l|l|l|}
\hline S0 & S1 & Output Frequency Scaling (fo) \\
\hline L & L & Power Down \\
\hline L & H & $2 \%$ \\
\hline H & L & $20 \%$ \\
\hline H & H & $100 \%$ \\
\hline
\end{tabular}




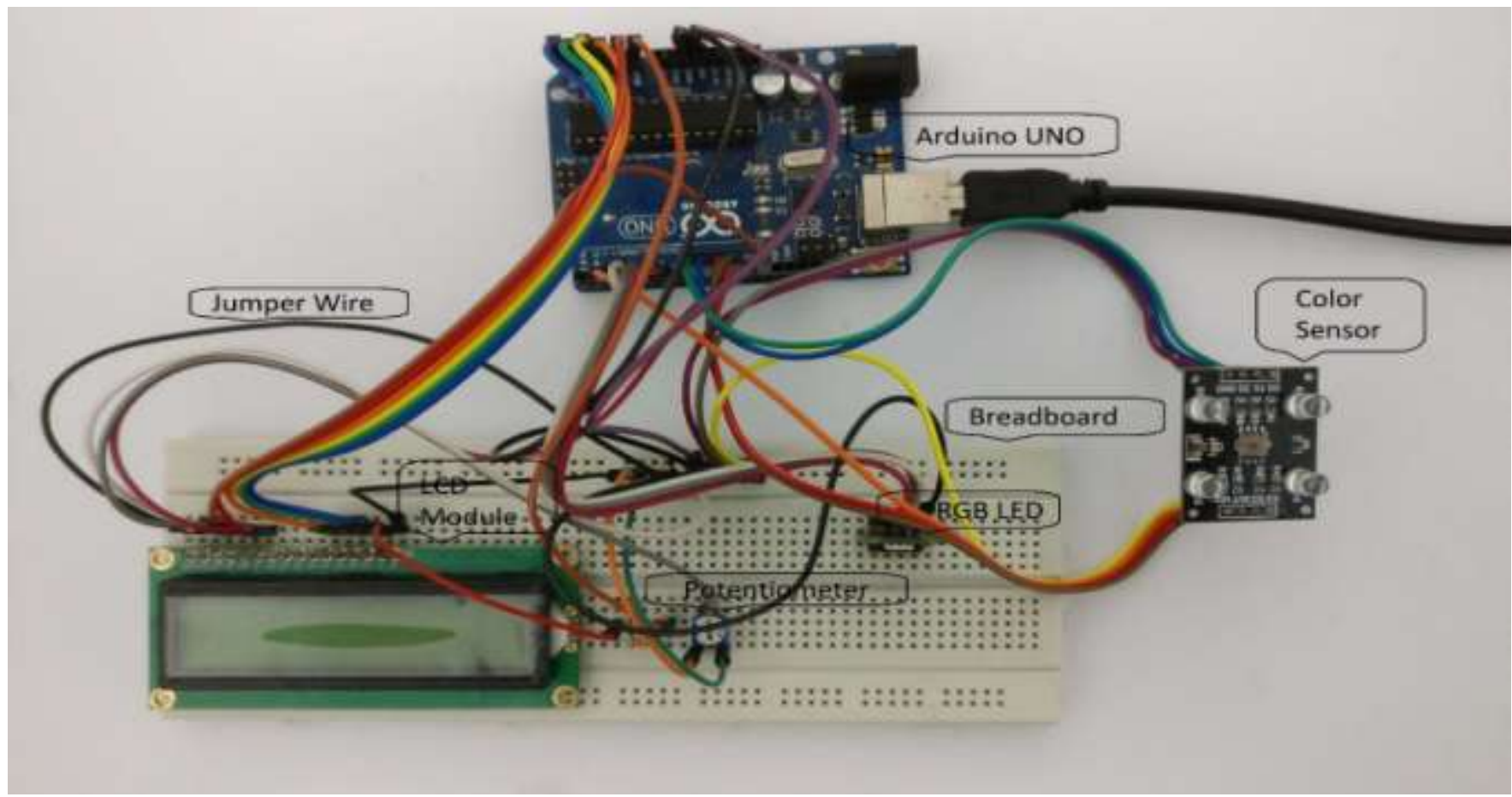

Fig 6: Electronic CircuitDiagramfor theSystem

Fig. 6 shows the circuit diagram of the RGB color detector using TCS3200.

RGB color detector using TCS3200.

Simplest Arduino codes compare the real values of $\mathrm{R}, \mathrm{G} \&$ $\mathrm{B}$ read by the sensor with predefined $\mathrm{R}, \mathrm{G} \& \mathrm{~B}$ values. The problem is that the $\mathrm{R}, \mathrm{G} \& \mathrm{~B}$ values read by the sensor, for a given object, depends on the relative position of the sensor from the object and on the external light conditions. So, a given object can be or cannot be recognized by the sensor in function of its relative position from the sensor [10]. Moreover, objects with very close colors to each other will be not recognized. To prevent such situations we must teach the sensor what are the range of variation of each parameter $\mathrm{R}, \mathrm{G}$ and $\mathrm{B}$, for a given color and its nuances, in function of its relative position from the sensor and different external light conditions. We call this action "calibration" in our code. For instance, when we want to teach the sensor to recognize the Color 1, we proceed as follows. First we reset the sensor, (we push the button "Reset button"), then we place a given object in the front of the sensor, we start the calibration process, (we push the "Rbutton"), and we change the position of the object and the external lighting conditions in order to reproduce the real conditions of measurements. We repeat the procedure with objects with very close colors to each other. So the sensor learns and memorizes the domain of variation of parameters R, G \& B for object of Color 1 and nuances of Color 1 . We repeat the procedure for three colors, Color 2 and Color 3. After that the sensor is ready for use. Each time when an object is placed in front of the sensor it will display the percentages of R, G \& B.

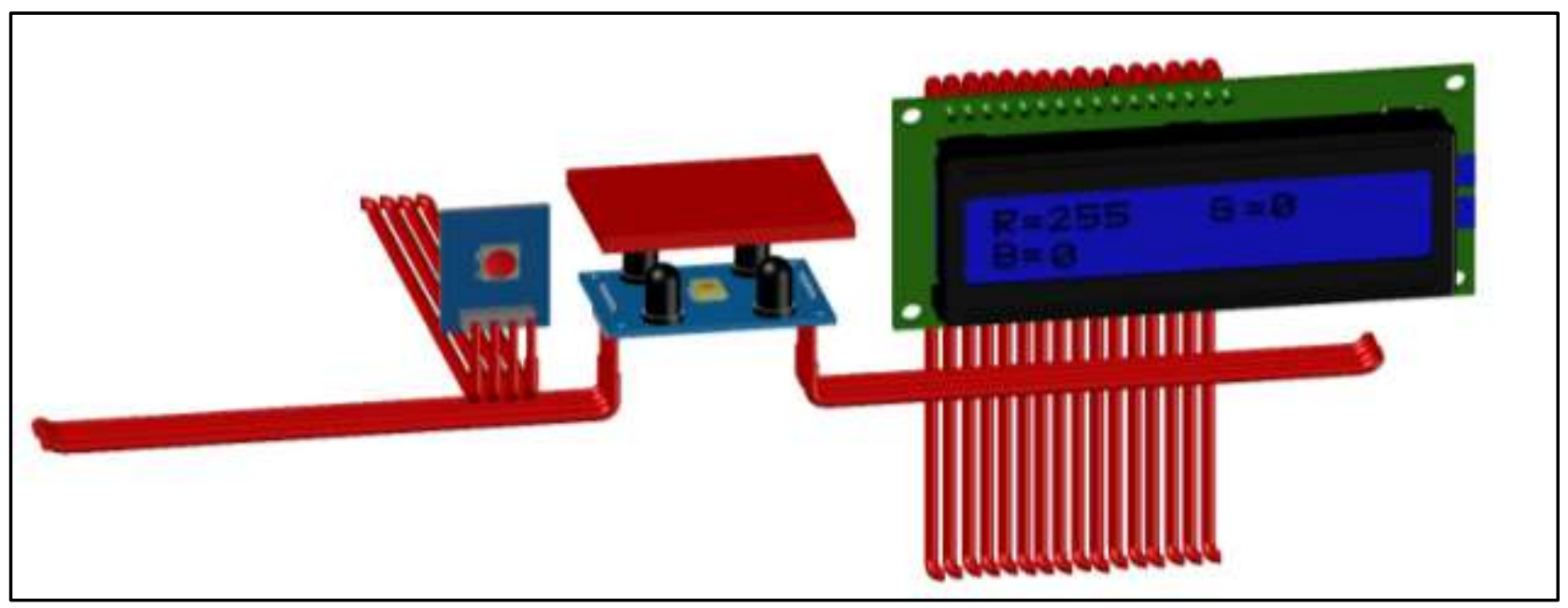

Fig 7: Schematic diagram of the system 
A Schematic diagram is shown in Fig. 7.When red color shade is kept near the sensor, it automatically detects the color with the help of photodiode arrays and then RGB color intensity value is displayed in Arduino serial monitor window and $16 \times 2$ LCD Module [11].
At the same time, a red LED glows in the RGB LED module. Similarly, the remaining two colors (green and blue) are shown in Arduino serial monitor window and the respective color LED glows in RGB LED module.A working model of the system is shown in Fig. 8.

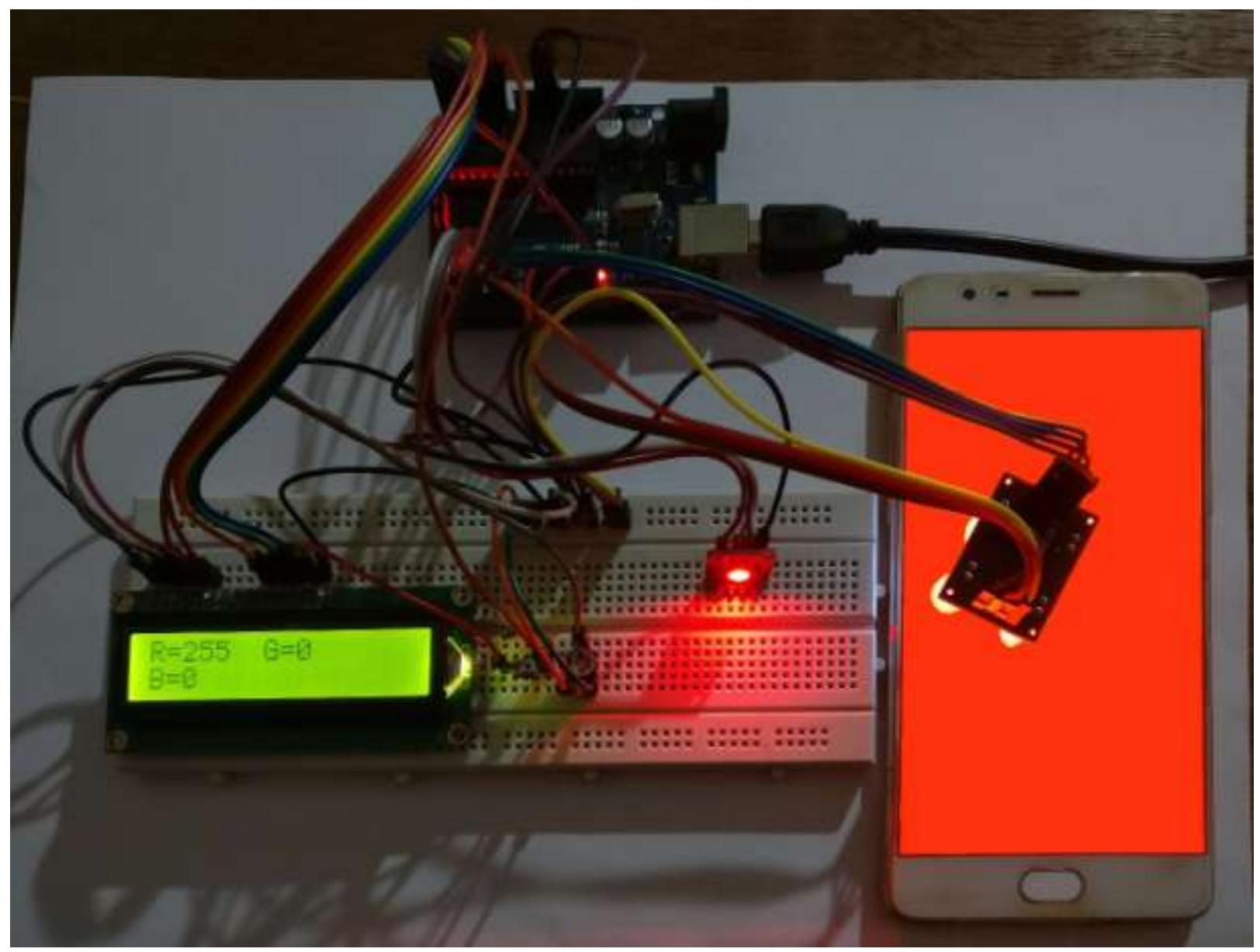

Fig. 8. Working Model of the System

\section{MATERIALSUSED\&THEIR APPLICATIONSIN PRESENT WORK}

Table 3: Materials Used \& their Function

\begin{tabular}{|c|c|c|l|}
\hline S.no & Item & Quantity & \multicolumn{1}{c|}{ Application } \\
\hline 1. & Arduino Board (UNO) & 1 & $\begin{array}{l}\text { The Arduino Uno R3 is a microcontroller board based on } \\
\text { the ATmega328. It has 14 digital input/output pins (of which 6 can be } \\
\text { used as PWM outputs), 6 analog inputs, a 16 MHz crystal oscillator, a } \\
\text { USB connection, a power jack, an ICSP header, and a reset button. }\end{array}$ \\
\hline
\end{tabular}




\begin{tabular}{|c|c|c|c|}
\hline 2. & Color Sensor (TCS3200) & 1 & $\begin{array}{l}\text { TCS3200 is a programmable color -to-frequency converter that } \\
\text { combines configurable silicon photodiodes and a current-to-frequency } \\
\text { converter on a single monolithic CMOS integrated circuit. The output } \\
\text { is a square wave ( } 50 \% \text { duty cycle) with frequency directly } \\
\text { proportional to light intensity.The TCS3200 reads an } 8 \times 8 \text { array of } \\
\text { photodiodes. }\end{array}$ \\
\hline 3. & RGB SMD LED Module & 1 & $\begin{array}{l}\text { RGB SMD LED is a surface mount device module, which consists of } \\
\text { a full color LED made by R, G, B. Primary colors (red / blue / green) } \\
\text { strength can be adjusted using three-pin PWM voltage input in order } \\
\text { to achieve full color mixing effect. }\end{array}$ \\
\hline 4. & $\begin{array}{l}\text { LCD Display Screen } \\
\text { (16 pin) }\end{array}$ & 1 & $\begin{array}{l}\text { The Liquid Crystal library allows you to control LCD displays. } \\
\text { The LCDs have a parallel interface, meaning that the microcontroller } \\
\text { has to manipulate several interface pins at once to control the display. } \\
\text { The process of controlling the display involves putting the data that } \\
\text { form the image of what you want to display into the data registers, then } \\
\text { putting instructions in the instruction register. }\end{array}$ \\
\hline 5. & Potentiometer (10k ohm) & 1 & $\begin{array}{l}\text { A potentiometer is a used for adjustment, tuning and calibration in } \\
\text { circuits. It can be used as a variable resistor. It is normally mounted } \\
\text { on PCB and adjusted by using a screwdriver. }\end{array}$ \\
\hline 6. & Breadboard & 1 & $\begin{array}{l}\text { A breadboard is a solder less device for temporary prototype with } \\
\text { electronics and test circuit designs. Most electronic components in } \\
\text { electronic circuits can be interconnected by inserting their leads or } \\
\text { terminals into the holes and then making connections through wires } \\
\text { where appropriate. }\end{array}$ \\
\hline 7. & JumperWire & $\begin{array}{c}\text { As Per } \\
\text { Use }\end{array}$ & $\begin{array}{l}\text { A jump wire (also known as jumper, jumper wire, jumper cable, } \\
\text { DuPont wire, or DuPont cable - named for one manufacturer of them) } \\
\text { is an electrical wire or group of them in a cable with a connector or pin } \\
\text { at each end. }\end{array}$ \\
\hline
\end{tabular}




\section{PART OF WORKING CODE OF PRESENT WORK}

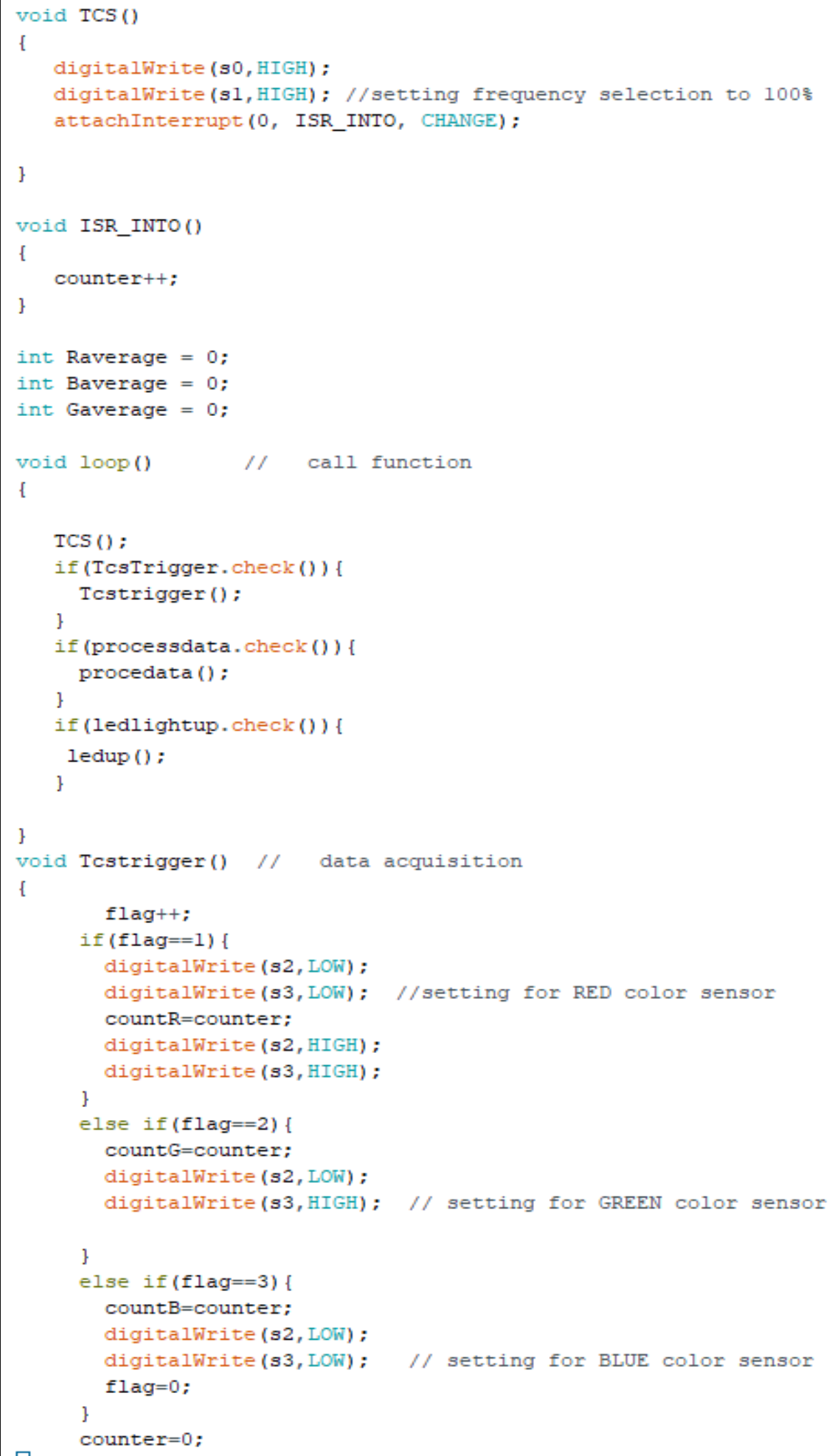

Fig 7: Working Code 


\section{CONCLUSION}

The present work on the development of the color detection and analyzing system has been tested and can be used for developing desired shades of color as per the sample shade. This may prove to be useful for paint and dye industries. The developed system determines the percentage of Red, Blue and Green colors in the sample shades. This information was further used to calculate the amount of these colors in any mixture of the given sample.

\section{REFERENCES}

[1] Daud, N.B., "Application of Colors Sensor In An Automated System", Technical University Malaysia, Melaka,May 2007.

[2] "Reflective Color Sensing with Avago Technologies" RGB Color Sensor, 2005.

[3] "Understanding Avago Technologies RGB Color Sensors", Publication number: AV01-0444EN.

[4] Texas Advanced Optoelectronic Solutions (TAOS009 - July 2009)

[5] Color Sensor-Digital Color Sensor IC-TCS3414 Ams. (Accessed on 16 February 2014).

[6] SensorWiki, Z., Acrob041 "Color Sensor TCS230"

[7] Saracoglu, O.G., Altural, H., Color Regeneration from Reflective Color Sensor Using an ArtificialIntelligent Technique. Sensors 2010, 10, 8363-8374.

[8] Pardo, P.J., Pérez, A.L., Suero, M.I., Chromatic characterization of a three - channel colorimeter using back-propagation neural networks. Rev. Sci. Instrum. 2004, 75, 2876-2879.

[9] D. Raja. 2016. Light Intensity Measurement Using LDR and AVR Microcontroller.

[10] RGB Color Codes Chart (n.d.)

[11] Assaad, M., Yohannes, I., Bermak, A., Ginhac, D., and Meriaudeau, F., 2014, Feb. 17. Design and Characterization of Automated Color Sensor System University of Bourgogne. 7(1): 1-12. 